\title{
Scientific Program and Abstracts for the Symposium on Neuropsychological Rehabilitation, Liechtenstein 31 July 2006 to 1 August 2006
}

DAY 1: MONDAY 31 JULY, 2006

\subsection{0-9.40: KEYNOTE INTRODUCTION}

\section{Current Challenges for Neuropsychological Rehabilitation}

B.A. Wilson

MRC Cognition and Brain Sciences Unit, Cambridge, UK

\begin{abstract}
A lthough we have come a long way in improving services for people with brain injury since modern rehabilitation began in World War I, there are still a number of challenges facing us at the beginning of the $21 \mathrm{st}$ century. This paper addresses six of them. First, we need to strengthen the link between basic cognitive neurosciences and rehabilitation as results from the former remain of limited use to those of us dealing with the practical problems faced by survivors of brain injury. Second, we need to ensure that findings from neuroimaging really benefit rehabilitation in more than just name. Third, we should collaborate more with the pharmaceutical industry in the expectation that a combination of drug and neuropsychological treatments will lead to better results than either treatment used alone. Fourth, we need to improve ways of evaluating rehabilitation, which will mean relinquishing dependence on traditional outcome measures that frequently fail to apprehend the real needs of patients and families. Fifth, we must learn to make more informed decisions when selecting treatments so that our choice of whether to attempt restoration of lost function or to teach compensatory strategies or to rely on environmental modifications is based on more than just intuition. Finally, we need to know how best to persuade health care purchasers to fund rehabilitation: how do we convince them that while rehabilitation is likely to be expensive initially, it will be cost effective in the long term?
\end{abstract}




\title{
9.20-11.00: SESSION 1 - ASSESSMENT
}

\subsection{0-10.00: Predicting the End of Post-Traumatic Amnesia at the Beginning of Rehabilitation Admission}

\author{
R. Tate, ${ }^{1}$ A. Pfaff ${ }^{2}$ and M. Perdices ${ }^{3}$ \\ ${ }^{1}$ Rehabilitation Studies Unit, University of Sydney, Australia \\ ${ }^{2}$ Brain Injury Rehabilitation Unit, Liverpool Hospital, Sydney, Australia \\ ${ }^{3}$ Department of Neurology, Royal North Shore Hospital, Sydney, Australia
}

\begin{abstract}
Rackground: Knowing in advance when a patient with traumatic brain injury is likely to emerge from post-traumatic amnesia (PTA) has many clinical advantages. We previously developed a regression equation to predict PTA duration using the first 5 days of PTA testing. This study aimed to refine the prediction using different predictor variables and a revised criterion for the end of PTA. Methods: A consecutive series of 84 inpatient rehabilitation patients was examined daily with the Modified Oxford PTA Scale. A series of regression equations was developed for predicting the length of time between the commencement of PTA testing and end of PTA using the revised criterion (first day scoring 12/12). Results: The best model for prediction comprised a single variable (sum of PTA scores for the first three days of testing), which accounted for $45 \%$ of the variance. Median discrepancy in days post-trauma between the predicted and actual times was -0.35 days (IQR 7.92). Large discrepancies occurred in 8/84 patients, most of whom had extended PTA durations ( $2-3$ months). Conclusion: Our regression model enables the clinician to reliably and easily predict duration of PTA within the first few days of testing the patient, thereby providing a practical adjunct for clinical care and management.
\end{abstract}

\subsection{0-10.20: Assessing Construct Validity of the European Brain Injury Questionnaire (EBIQ) Using Rasch Analysis}

A. Bateman and M. Horton

East Cambs and Fenland PCT, The Oliver Zangwill Centre, Princess of Wales Hospital, Ely, UK

Background: Studies of effectiveness of rehabilitation require measure-
ment tools that have established measurement properties that are appro-
priate for the patient group. Rasch analysis is an interesting method for
examining rating scale validity. Methods: Responses to the EBIQ from 226
people were entered into the analysis. The sample ( $72 \%$ male, median age
34 ) with acquired brain injury ( $1-10$ years prior to assessment) represented
consecutive referrals to Oliver Zangwill Centre. The EBIQ consists of 63
items requiring a rating of frequency of symptoms (cognitive, physical,
motivational impairments). There are three response categories for each item
(not at all, a little, a lot). Results: Seventeen items were excluded: 14 because
ChiSq P values $<.05$ and three further items were removed as they displayed
significant uniform DIF by gender (crying easily, irritability, finding your
way). After exclusion of these, the remaining 49 items yielded an overall fit
to the Rasch model (Total item $\chi^{2}=159.6$, P $=.10$ ). Principal components
analysis was used to examine local independence. No significant differences
were found between person estimates given by the subsets when compared to
person estimates given by the full 47 -item scale. This supports the assump-
tion of unidimensionality of the scale. Visual inspection of a plot of targeting
revealed a good spread of items across the full range of respondents' scores
indicating that the items provide a good operational measurement range for 
difficulties experienced by people with brain injury. Conclusion: This analysis demonstrated the scale does not work as a 63 -item scale. Further work on proposed subscales is underway. The analysis provides a platform for further parametric analysis of impact of rehabilitation. A score based on 46 EBIQ items meets the expectations of Rasch model and can be considered a valid unidimensional scale for assessing personal experience of brain injury.

\subsection{0-10.40: Socioemotional Processing: A Comparison Between Children With Brain Injury and Noninjured Controls}

\section{J. Tonks, W.H. Williams, I. Frampton, P.J. Yates and A. Slater}

School of Psychology, University of Exeter, Washington Singer Laboratories, Exeter, UK

$O$ verview: The ability to interact with others is dependent upon a range of cognitive and emotional systems operating effectively; systems which plan and organise behaviour, and systems that may function at the basic level of analysis of stimuli, such as recognising whether someone else is happy, angry or sad. Research findings previously reported in the literature have been integrated into a dissociable schematic, which offers a novel representation of the subcomponents of the emotion processing system. We advocate that a proportion of lasting emotional and social communication difficulties, which are frequently observed among children who have suffered a traumatic brain injury, are a result of compromise to emotion processing systems in the brain. These systems function in reading emotion in others from analysis of eyes, face expression, or emotional vocal prosody for example. Little is known about how brain injury affects the development of these skills. Methods: In the current study, novel and adapted measures of emotion processing were used in requisite tasks of spontaneous socioemotional judgment, reading emotion from voices, eyes and faces. These were used, in conjunction with a battery of tests of cognitive function, in gathering data from a cross-section of 20 children aged between 9 and 17 with acquired brain injuries. Performance levels were compared against the normative data from 67 matched 'healthy' children. Questionnaires were used as a measure of socio-emotional behaviour. Results: ABI children were significantly more impaired in emotion processing. Attention switching and visual discrimination skills were found to predict ability to read facial expression in both groups. Specific emotion reading skills relating to facial or vocal expression were found to predict behaviour disturbance. Many of the elements of emotion processing tested were found to worsen at 11 years old following injury — indicating a delay in the acquisition of skills, while in noninjured controls there was improvement. Conclusions: Postbrain-injury social and emotional difficulties may result from the delay in acquisition of emotion recognition skills at 11 years old, identified. 


\title{
10.40-11.00: Constructing Selves After Acquired Brain Injury: Capturing Identity Change in Neuropsychological Rehabilitation Using a Personal Construct Approach
}

\author{
K. Psaila, ${ }^{1,2}$ F. Gracey, ${ }^{1}$ J. O’Dell, ${ }^{1}$ J. Cope, ${ }^{1}$ S. Mohamed ${ }^{1,2}$ and S. Palmer ${ }^{1}$ \\ ${ }^{1}$ The Oliver Zangwill Centre, Princess of Wales Hospital, Ely, UK \\ ${ }^{2}$ Cambridgeshire and Peterborough Mental Health Trust, UK
}

\begin{abstract}
Changes in self-concept and comparison of pre- and postinjury selves have been recognised in the literature on the consequences of acquired brain injury. Application of these research findings to rehabilitation activity and outcome measurement has not been reported. This paper describes use of a personal construct approach integrating personal perspectives and clinically useful measurement within group rehabilitation activity. We present initial data from a broader study of identity change in holistic rehabilitation that addresses the question of how people see or 'construct' themselves postinjury. Eighteen rehabilitation clients with nonprogressive ABI engaged in structured group exercises to discuss their pre, post and ideal 'selves'. This process yielded 113 bipolar constructs in total, from 5 groups of between 2 to 5 participants. These were analysed using thematic analysis. Emergent themes highlighted constructs relating to identity, emotion, skills, abilities and roles. It is concluded that the exercise of comparison of 'selves' yields meaningful constructs covering a range of issues. Support is found for the notion that identity, emotion, and social factors play a significant part in postinjury adjustment. The group exercise may be of use in eliciting discussion of identity change in group-based rehabilitation, and for developing personal rating scales for measuring change.
\end{abstract}

\subsection{0-11.40 MORNING TEA}

\subsection{0-12.40: SESSION 1 (CONT.) - ASSESSMENT}

\subsection{0-12.00: Evidence for a Relationship Between Alertness and Leftward Inattention}

A. Dove, C. Dodds, M. George, J. van Belle and T. Manly

MRC Cognition and Brain Sciences Unit, Cambridge, UK

The current study investigated the hypothesised link between alertness and 1 leftward inattention in neglect using a modified version of the star cancellation task of the Behavioural Inattention Test (BIT) battery. Unilateral spatial neglect is a striking, common and debilitating condition in which patients have difficulty in detecting or responding to information from one side of space, usually the left. The severity of neglect shown by individual patients can vary considerably from one time to another and it has been hypothesised that changes in alertness might be one source of this variability. To examine this, three patients were presented with several A4 sheets of paper on which small stars were distributed among larger stars, words and letters and were required to mark each small star with a pen. Patients were expected to miss more stars on the left of the sheet when they were less alert. In order to investigate this prediction we measured the time taken to cancel the first 30 stars and used this as a measure of how each patient's level of subjective alertness fluctuated from 
one sheet to the next. As predicted, there was a correlation between this measure and the eventual degree of rightward spatial bias.

\title{
12.00-12.20: The Pint Test
}

E. Greenfield, J. Evans, H. Emslie and B.A. Wilson

MRC-Cognition and Brain Sciences Unit, Cambridge, UK

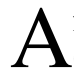
fter a brain injury (BI) many people have a problem with dual tasking (DT), which has serious repercussions on many aspects of their life, affecting activities of daily living, returning to work and leisure. There are few tools available to enable clinicians to assess DT performance. As part of the development of a new battery of tests of divided attention and DT (The DivA), we designed a test that has a high ecological validity and is sensitive to difficulties in combining cognitive and motor tasks. The Pint Test involves a primary condition walking 5-metre lengths for 2 minutes carrying a full glass of water (inside a larger glass), the aim being to spill the minimum whilst walking as many lengths as possible. In a second condition, the added demand of conversing is included. The two conditions are compared in terms of the volume of water spilt per length walked in order to derive an index of cognitive-motor DT performance. We will present performance data for 200 controls and 100 people with BI. Some BI people, under DT conditions, tend to be more stimulus driven, sacrificing the more automatic, learned motor action of walking in favour of the more attentionally demanding tasks, whilst others perform surprisingly well in a demanding task. Possible reasons will be discussed.
\end{abstract}

\subsection{0-12.40: Endovideolaryngoscopy in Phoniatricians Practice}

M. Vukašinovi and D.P. Stankovi

Clinical Center of Serbia, ENT\&MFS Institute, Phoniatrics Dpt., Beograd, Serbia

Q ndovideolaryngoscopy is the obliged evaluation tool in everyday prac-

Etice in our Phoniatrics Department with 10,000 patients annually. Standardised protocol in management of broad spectrum of vocal pathology is useful in clinical, scientific and educational evaluation of patients from the first interview till the end of the treatment. Using contemporary computerised multidimensional analysis of stroboscopic image, we are approaching an optimum evaluation set of any kind of interpersonal communication disorder.

12.40-14.00 LUNCH 


\subsection{0-15.20: SESSION 2 - INTERVENTIONS}

\subsection{0-14.20: Learning Face-Name Associations in Early-Stage Dementia: Comparing the Effects of Errorless Learning and Effortful Processing}

L. Clare and J. Dunn

School of Psychology, University of Wales Bangor, Bangor, UK

Some recent studies suggest that errorless learning principles may be bene$\checkmark$ ficial in memory rehabilitation for people with dementia, while others indicate that effortful processing may be more important. The present study compared the effects of four different learning techniques, varying in level of effort required and number of errors elicited, on free recall, cued recall and recognition of novel and previously known associations among people with early-stage dementia. Ten participants with a diagnosis of early-stage Alzheimer's, vascular or mixed dementia learned novel and previously familiar face-name associations with four techniques - vanishing cues, forward cues, target selection, and paired associate learning — in a within-subjects design. All conditions produced significant learning for both novel and familiar associations. There were no significant differences between conditions, although mean scores were slightly higher for errorful conditions. Reducing errors did not produce any benefits. Enhancing level of effort had no significant effects for familiar associations, but high-effort conditions were significantly more effective than low-effort conditions in facilitating cued recall of novel associations. The results confirm that memory rehabilitation techniques can produce significant benefits, but do not support the view that error reduction during learning facilitates greater improvement. Effort enhancement may be more important, especially when learning novel associations.

\subsection{0-14.40: Errorless Learning: A Comparison With Other Memory Rehabilitation Techniques}

K. Hodder and C. Haslam

School of Psychology, University of Exeter, Exeter, UK

Three promising techniques used in memory rehabilitation are errorless 1 learning, vanishing cues, and spaced retrieval. However, their effectiveness has rarely been compared. This research compared these techniques, both in isolation and in combination. A dual task was developed to reduce the memory capacity of healthy adults and make it comparable with that of memory-impaired patients. We found that under dual-task conditions healthy adults generally performed at the level of patients with mild to moderate memory impairment. The three techniques were then used to examine learning of object- and face-name associations in healthy adults under standard and dual task conditions. Results showed that errorless learning was effective, as reported in previous research. However, vanishing cues, both alone and in combination with errorless learning, was less effective than errorless learning alone. Preliminary findings with spaced retrieval suggest that the technique is effective alone, and offers potential for increased effectiveness when combined with errorless learning. Importantly, the pattern of results did not appear to change under dual task conditions, suggesting memory-impaired patients may respond similarly to the techniques. This is currently being investigated in a memory-impaired population. These results encourage a more targeted approach to the design of memory rehabilitation programs. 


\title{
14.40-15.00: Forgot to Walk the Dog? An Experimental Single Case Study of Carer-Facilitated Errorless Learning Following TBI
}

L. Campbell, ${ }^{1}$ F.C. Wilson, ${ }^{2}$ J. McCann, ${ }^{3}$ G. Kernahan ${ }^{4}$ and G.R. Rogers ${ }^{5}$

${ }^{1}$ Occupational Therapy Department, Department of Rehabilitation Medicine, Royal Group of Hospitals Trust, Belfast, Northern Ireland

2 Department of Clinical Neuropsychology, Joss Cardwell Centre, Belfast, Northern Ireland

${ }^{3}$ Department of Rehabilitation Medicine, Royal Group of Hospitals Trust, Belfast, Northern Ireland

${ }^{4}$ School of Nursing, University of Ulster, Jordanstown, Northern Ireland

${ }^{5}$ School of Communication, University of Ulster, Jordanstown, Northern Ireland

Primary Objective: The objective of this clinically based study is to investigate if errorless learning applied by carer(s) in an everyday setting can reduce the frequency of everyday memory problems following severe traumatic brain injury (TBI). Research design: Multiple baseline single-case experimental design. Methods and Procedures: A patient with severe memory impairment, 6 years post-TBI, was recruited via an outpatient neurorehabilitation clinic. ABA analysis was conducted using daily frequency counts of everyday memory problems as an index of change. Experimental Intervention: Intervention within an errorless learning paradigm was delivered by the patient's carer, aimed at reducing the occurrence of identified everyday memory problems. Patient generated cues were used and the carer was guided in treatment implementation by an Occupational Therapist. Main Outcomes and Results: Incidence of frequently occurring memory lapses reduced significantly $(p<.0001)$ and this was maintained at 3 months postintervention. Conclusion: This study highlights the potential clinical value of errorless learning, using patient generated cues applied by carers within an everyday setting.

\subsection{0-15.20: Is Memory Rehabilitation Possible in Older, Densely Amnesic Adults? A Detailed Case Study of a Post-Encephalitic Patient Using Errorless Learning and a Personal Digital Computer}

\author{
Ashok S Jansari, ${ }^{1}$ Catherine Gardner ${ }^{1}$ and Barbara Wilson ${ }^{2}$ \\ ${ }^{1}$ School of Psychology, University of East London, London, UK \\ 2 MRC-Cognition and Brain Sciences Unit, Cambridge, UK
}

Jeuropsychological research has shown that both procedural (Corkin, 1 1968) and implicit memory remain intact in patients with amnesia (e.g., Schacter, 1987; Graf et al., 1984). Research has capitalised on this to develop rehabilitation paradigms for patients using a range of methods and devices such as NeuroPage (NP; e.g., Wilson et al. 2001). In a single case study, Wilson et al. (1997) suggested that a younger age at the time of amnesic insult was one strong predictor of successful rehabilitation. We report the case of a 62-year-old densely amnesic postencephalitic patient, NP using an errorless learning method (Baddeley \& Wilson, 1994) in conjunction with an electronic personal digital computer (PalmTop) to remember to perform a series of activities of daily living. Despite NP's age, the regime proved to be very successful with significant gains following training. The bearings that this finding has on rehabilitation attempts with other groups of patients are discussed. 


\title{
16.00-17.20: SESSION 2 (CONT.) - INTERVENTIONS
}

\subsection{0-16.20: External Alerting Strategies for the Rehabilitation of Executive Dysfunction in Acquired Brain Injury}

\author{
J. Fish, J.J. Evans, M. Nimmo, E. Martin, A. Bateman, B.A. Wilson and T. Manly \\ MRC Cognition and Brain Sciences Unit, Cambridge, UK
}

\begin{abstract}
$\mathrm{T}$ his study investigated the effects of using an alerting strategy on the prospective memory performance of a group of adults $(n=21)$ with acquired nonprogressive brain injury and prospective memory problems resulting from executive dysfunction. Frequently, failures of prospective memory can be attributed to attentional or executive disruption, for example due to impaired planning, strategy application, resistance to distraction, or more globally, 'goal neglect' (Duncan et al., 1996). Participants received training in the use of external alerts to prompt consideration of overall plans, goals, and other 'things to do' (Goal Management Training; Robertson, Levine, \& Manly, in preparation). The efficacy of the strategy was examined with a task requiring participants to make calls to a voicemail service at four times each day for ten days. Task content was encoded using errorless learning to minimise retrospective memory-based failures. On five randomly selected days, eight text messages reading simply 'STOP!' were sent to participants' mobile telephones, but crucially not within an hour of designated target times. Despite there being no direct link to the experimental task in terms of either time of receipt or cue content, striking benefits were seen in task performance on cued days compared to days without cues. This constitutes encouraging evidence that alerting strategies are useful in helping remediate problems resulting from executive dysfunction. It is proposed that this benefit results from enhanced efficiency of goal management via increased monitoring and consideration of current and future actions and goals.
\end{abstract}

\subsection{0-16.40: Using a Paging System in the Rehabilitation of Four Encephalitic Patients}

\author{
H. Emslie
}

MRC Cognition and Brain Sciences Unit, Cambridge, UK

$\mathrm{M}$ emory and executive problems following encephalitis are common yet there are few published papers on the successful rehabilitation of such patients. We recently demonstrated (Wilson, Emslie, Quirk, \& Evans, 2001; Wilson, Emslie, Quirk, Evans, \& Watson, 2005) that a paging system could reduce everyday memory and planning problems for people with nonprogressive brain injury. Among the 143 patients who participated in the 2001 study were 4 people who had survived encephalitis. Their results are reported here. During a 2-week baseline, the successful task achievement of our four clients ranged from $2 \%$ to $81 \%$. Each then received a pager for 7 weeks and task achievement was documented in weeks 6 and 7. All were significantly more successful with the pager than they had been at baseline with success rates ranging from $45 \%$ to $96 \%$. Five weeks after returning their pagers they were monitored once more. One of the encephalitic patients failed to achieve any of his target tasks, returning to baseline level, the other three dropped back a little but were still much more successful than at baseline. It is concluded that the paging system significantly reduces everyday memory and planning problems of patients with encephalitis. 


\title{
16.40-17.00: Learning and Usability of Electronic Aids to Daily Living After Acquired Brain Injury
}

\author{
I.-L. Boman, ${ }^{1,2}$ K. Tham, ${ }^{2}$ A. Granqvist, ${ }^{1}$ A. Bartfai ${ }^{1}$ and Hemmingsson ${ }^{2}$
}

${ }^{1}$ Rehabilitation Medicine University Clinic, Danderyd Hospital, Stockholm, Sweden

${ }^{2}$ Division of Occupational Therapy, Neurotec Department, Karolinska Institutet, Huddinge, Sweden

$\mathrm{T}$ This pilot study was the first study of electronic aids to daily living (EADL) where the users actually stayed for a longer period of time in apartments equipped with EADL in a new building in the inner city area of Stockholm, Sweden. The purpose was to study the ability of persons with memory impairments after acquired brain injury to learn and use EADL and to describe change in functioning and quality of life. Method: Eight participants stayed in two apartments equipped with a set of basic and advanced EADL between 4 to 6 months during an intervention time of 2 years. The method of errorless learning was used to teach the participants to use EADL. Ability to learn to use EADL was measured by structured observations. Function and quality of life were assessed with self-rating questionnaires. Results: Results showed that the participants learned to use EADL. They perceived that EADL was very useful and easy to learn. Occupational performance and satisfaction with occupational performance and quality of life was improved. Conclusion: The result shows that EADL may play an important role to facilitate everyday activities and improve satisfaction with occupational performance and quality of life for people with memory impairments.

\subsection{0-17.20: The Use of Sensecam as a Pictorial Diary to Improve Autobiographical Memory in a Limbic Encephalitis Patient With Amnesia: A Preliminary Report}

\author{
E. Berry, L. Williams, K. Wood, N. Kapur and B.A. Wilson
}

R3 Neurosciences, Addenbrooke's Hospital, Cambridge, UK

$\mathrm{T}$

This case study describes the use of a wearable camera, SenseCam, which automatically captures several hundred images per day, to aid autobiographical memory in a patient, Mrs B, with marked amnesia following limbic encephalitis. Mrs B's severe memory impairments led to anxiety and a loss of confidence in social situations. By using SenseCam to record personally experienced events, we hoped that the SenseCam pictures could be used as a pictorial diary to consolidate and cue her autobiographical memories. After a period of time wearing SenseCam, she plugged the camera into a standard PC which downloaded the recorded images and allowed them to be viewed at speed, like watching a movie. In the control condition a written diary was used to verbally record and remind her of autobiographical events. After viewing SenseCam images, Mrs B was, in the majority of cases, able to recall approximately $90 \%$ of the event. Retention of events was maintained in the longer term, many months after the event, and without looking at the SenseCam images for 2 months. As a result, Mrs B's subjective levels of anxiety reduced, and her confidence increased. By contrast, after using the written diary, Mrs B was able to remember on average $40 \%$ of an event 2 weeks after it occurred. A range of factors may have enabled Mrs B to recall her memories so effectively using SenseCam, and various hypotheses are discussed. The benefits and drawbacks of using SenseCam with brain-injured patients are also considered, as are SenseCam's potential further applications in aiding therapy and rehabilitation. 


\title{
09.20-11.00: SESSION 2 (CONT.) - INTERVENTIONS
}

\subsection{0-9.40: A Case for Continuing Rehabilitation: Improving Chronic Naming Abilities in a Brain-Injured Client}

C. Keohane and L. Prince

The Oliver Zangwill Centre, Princess of Wales Hospital, Ely, UK

\begin{abstract}
Communication difficulties following acquired brain injury vary greatly in type and degree of difficulty resulting in a diverse range of interventions by speech and language therapists. The purpose of this study was to determine whether Constraint Induced Aphasia Therapy (Pulvermuller et al., 2001) would be more effective than conventional therapy techniques as a treatment for a client with a chronic naming disorder. In addition the question arose as to whether changes in the client's naming ability were due to intensity of treatment rather than as a result of the treatment method alone. A single-case experimental design was employed with phases that included alternating and combining different treatment methods and intensity of treatments during a 6-month neuropsychological rehabilitation program. Pretreatment and posttreatment measurements were taken using standardised tests as well as informal qualitative assessment. Statistical analyses including chi-square analyses, were used to analyse pre- and posttreatment results. Improved naming ability during the more intensive phase suggested that intensity of treatment, involving a conventional treatment approach, proved most successful for this client despite the chronic nature of the difficulty. The implications for clinical practice are that alongside factors of the holistic rehabilitation approach that are believed to have impacted on performance, specific levels of intensity and individualised treatment techniques are required to make some impact.
\end{abstract}

\subsection{0-10.00: Training Face Identification in Prosopagnosia}

\section{B.-K. Dewar and B.A. Wilson \\ MRC Cognition and Brain Sciences Unit, Cambridge, UK}

Drosopagnosia is the inability to recognise people from face alone, although recognition from other modalities remains intact (Francis, Riddoch, \& Humphreys, 2004). It has proved notoriously difficult to treat. We describe the case of VO who presented with impaired face recognition of familiar people following herpes simplex encephalitis. Photographs and semantic information were collected of family and friends that VO had difficulty recognising. Following multiple baseline assessment, eight faces were selected for training. Face identification was trained with the use of a mnemonic and the errorless learning paradigm of expanded rehearsal. Two faces were trained at weekly sessions, in addition to home practise. Recall of all faces was tested at the beginning of each session. Maintenance and generalisation of learning were also assessed. VO was able to correctly name the faces following training. There was evidence of generalisation of learning to different (profile) photographs and learning was maintained in the absence of practise. Results are discussed with respect to semantic relearning and generalisation. Methodological issues of treatment of face and person identification impairments are also discussed. 


\title{
10.00-10.20: A Group Treatment Program for Mild Acquired Cognitive Dysfunction (MACD)
}

\author{
A. Bartfai \\ Rehabiliteringsmedicinska Universitetskliniken, Stockholm, Sweden
}

$\mathrm{T}$ he purpose of the study was to develop a program for treatment of longterm sequelae of mild acquired cognitive dysfunction (MACD) of different aetiologies in a group setting according to principles of neuropsychological rehabilitation. Group treatment was selected to create a social milieu, to reconstruct creative situations where cognition and emotion interact, to share experiences and to increase 'steam' during exercises. Aims were to improve coping strategies, identify acquired cognitive dysfunction, their emotional consequences, and to increase awareness of and motivation to change behaviour and to learn new strategies. The program was designed as an explicit model for the participants about how to organise daily schedules, how to observe periods of changes in capacity, how to create structure and benefit from it. Generalisation was facilitated in external tasks, 'homework' and by discussing the results in the group. The results indicated a significant improvement in ratings of quality of life, significant increase in self-control and a trend to decreased anxiety according to the Psychological Well Being Scale and significantly improved recall and the Buschke Selective Reminding Test. At the 3 months' follow-up 91\% of participants were very satisfied with the group treatment program.

\subsection{0-11.00: SESSION 3 - REHABILITATION PROVISION AND OUTCOME}

\subsection{0-10.40: Effectiveness of Vocational Rehabilitation Following Acquired Brain Injury: Preliminary Evaluation of a UK Specialist Rehabilitation Program}

L. Murphy

Rehab UK London Brain Injury Centre, London, UK

Primary objective: To report the outcome of 232 clients undertaking Rehab UK's Vocational Rehabilitation Programme, over 3 consecutive years, in three centres across the UK. Methods and procedures: Data regarding demographic characteristics of participants, type and severity of acquired brain injury were collected on admission to the programme. The duration of the vocational program and occupational status on discharge is also reported. Interventions: A description of the vocational program is given, including selection and admission criteria, main components of the program, monitoring and evaluation frameworks. Results: $41 \%$ of participants were discharged into paid competitive employment, with a further $16 \%$ gaining voluntary work and $15 \%$ taking up mainstream training or education. The remaining $28 \%$ were referred to other services or withdrew from the program. Severity of injury and type of injury were not related to outcome. Movement across the labour market from preinjury to postrehabilitation is presented. Conclusions: Vocational rehabilitation, offering educational and experiential learning opportunities, is effective in enabling participants with severe acquired brain injuries to return to paid employment. 


\title{
10.40-11.00: Cost Outcomes for Neurobehavioural Rehabilitation: A Multicentre Clinical and Economic Appraisal
}

\author{
A. Worthington, S. Matthews, Y. Melia and M. Oddy \\ West Heath House, Birmingham, UK
}

\begin{abstract}
A multicentre cost-outcome study was undertaken to evaluate the effectiveness of neurobehavioural rehabilitation by a leading independent service provider in the UK. A prospective cohort of 135 severely braininjured adults contributed data at admission and discharge relating to type of residence, amount and type of care, functional independence, engagement in constructive occupation and participation in social roles. Follow-up data were obtained independently for 62 patients at an average of 18 months postdischarge. Data were analysed in terms of social outcomes and support costs. Improvements were evident in type of placement, levels of care, functional ability, productive occupation and participation. Although reemployment rates were low for this severely injured cohort comparison with preinjury occupation suggests that a significant minority had little constructive occupation premorbidly. Cost savings were calculated, taking into account treatment costs, and subject to sensitivity analysis over the projected lifetime of the cohort. The results demonstrated significant savings in support costs with rehabilitation, with the greatest savings being made when treatment if begun within the first year of injury. Taking account of these savings we conclude that, regardless of the initial cost, rehabilitation generally pays for itself within 2 years.
\end{abstract}

11.00-11.40 MORNING TEA

\subsection{0-12.40: SESSION 3 (CONT.) - REHABILITATION PROVISION AND OUTCOME}

\subsection{0-12.00: Understanding and Coping: Family Members' Experience of Rehabilitation Services}

\section{S. Palmer}

The Oliver Zangwill Centre, Princess of Wales Hospital, Ely, UK

The crucial role families play in rehabilitation is increasingly acknowledged
in clinical practice and government targets. Although desirable, it is not
always possible for professionals to work collaboratively with family mem-
bers. This project explored how collaboration might be improved. Questions
addressed whether understanding of brain injury (UBI) or individual coping
style related to use of rehabilitation strategies. Family members' experiences
of helpful and unhelpful aspects of professionals were also explored. A mixed-
methods design was incorporated. Thirty-two participants completed mea-
sures of: UBI, mood, strategy use and coping style. Data were analysed using
correlations. Semistructured interviews explored experiences of collaboration.
All transcripts were analysed using top-down theme analysis for specific ques-
tions. Four were further analysed using Interpretative Phenomenological
Analysis to identify emergent themes. Results revealed no statistical relation-
ships between UBI and use of strategies, or coping style. However, qualitative
analyses revealed a complex relationship where UBI is crucial to collabora-
tion, and to use of strategies. A theme about expectations of services emerged,
which linked with dissatisfaction. Essential characteristics of professionals


were identified as empathy, honesty and competence. This project offers insight into perspectives of 32 family members. Suggestions for managing expectations, such as increasing information and opportunities to ask questions, are discussed.

\title{
12.00-12.20: Narrative Construction of Self and Personal Navigation After Head Trauma: The Practice of Holistic Neuropsychological Rehabilitation
}

\author{
P. Frommelt, W. Kühne and H. Grötzbach \\ Neuropsychology Department, Asklepios Klinik Schaufling, Schaufling, Germany
}

\begin{abstract}
$\boldsymbol{R}$ ackground: There has been some debate about the principles of neuro$\boldsymbol{B}_{\text {psychological rehabilitation after head trauma. However, the everyday }}$ neuropsychological practice has received little consideration. The question arises which concepts and practical interventions neuropsychologists use in their clinical routines. Our presentation describes the use of narratives and the concept of personal navigation in holistic neuropsychological rehabilitation. Methods: A qualitative analysis based on a description of interventions and of audiovisual recordings from an inpatient rehabilitation program. Results: The participants in a holistic rehabilitation program display a wide diversity of narratives of their past, actual and future selves. As a common theme for these narratives the concept of personal navigation (Sternberg \& Swift-Sperling, 1998) is useful. After the shipwreck they search for new directions in their lives. The metaphor of personal navigation ascribes the role of the captain to the patient and that of a pilot to the psychologist. Successful navigation requires from the captain adequate self-awareness of strengths and weaknesses as well as effective compensatory skills. How the participants strive to find direction and control in their personal navigation is demonstrated. In practice, the use of narratives on the personal navigation is helpful to restore acceptance and self-efficacy.
\end{abstract}

\subsection{0-12.40: Holistic Neuropsychological Rehabilitation and Conventional Rehabilitation - Psychosocial and Vocational Outcome}

W. Kühne, S. Zillinger, P. Frommelt and H. Grötzbach

Neuropsychology Department, Asklepios Klinik Schaufling, Schaufling, Germany

Rackground: In the Asklepios Rehabilitation Hospital Schaufling both $\boldsymbol{B}_{\text {holistic and conventional neuropsychological rehabilitation programs }}$ for brain-injured patients are offered. Self-awareness, cooperative behaviour, resilience and goal-orientation are regarded therapeutic key areas in holistic group-milieu treatment programs (Prigatano, 1999). The placement to the different treatments is mostly initiated by the insurance companies. We were interested in the psychosocial and vocational outcome for both treatments one to four years after discharge. Method: In a retrospective case controlled study 60 patients with severe head injury were included, 30 in the holistic and 30 in the conventional treatment programs. The time since discharge varies between one to four years. In addition to a telephone interview several standardised questionnaires were used (CIQ, HADS, MKS, HI-FI and so on). We also received data from the significant others. Results: The evaluation of the data has not yet been completed. The final results will be presented at the meeting. One of the first surprising results is the $50 \%$ rate of return to work in both groups. 


\title{
14.00-15.00: SESSION 3 (CONT.) - REHABILITATION PROVISION AND OUTCOME
}

\subsection{0-14.20: Rehabilitation in Retrospect: A 12- to 15-Year Follow-Up of Patients Completing a Post-Acute Brain-Injury Rehabilitation Program}

\author{
H. Svendsen and T.W. Teasdale \\ Centre for Rehabilitation of Brain Injury Department of Psychology, University of Copenhagen, \\ Copenhagen, Denmark
}

\begin{abstract}
$\mathrm{T}$ The Center for Rehabilitation of Brain Injury (CRBI) at the University of Copenhagen has provided post-acute holistic rehabilitation for adults with acquired brain injury for over 20 years. The CRBI program comprises elements designed to promote physical, cognitive, emotional and social development. As part of a 12- to 15-year follow-up study, 52 subjects who had completed the program at some time between 1989 and 1992 were asked to rank the program elements according to degree of importance which they perceived them to have had for for their development. Consistently, most highly rated was contact with other persons with brain injury. Individual work with a primary therapist and cognitive training were also rated highly. Group psychotherapy sessions were given a low rating, as were post-program follow-up meetings. The subjects were also asked to rate how much the rehabilitation program overall had meant for various aspects of their lives. Most highly rated was 'general quality of life' and 'help with cognitive problems'. Specific help with return to the labour market, and promoting family relations and leisure activities were rated lowest. The implications of these findings are discussed in relation to the subjects' demographic and medical characteristics.
\end{abstract}

\subsection{0-14.40: Goal Theory, Self-Regulation and Brain Injury Rehabilitation}

J.J. Evans ${ }^{1,2}$ and T. Hart ${ }^{3,4}$

1 University of Glasgow Section of Psychological Medicine, Glasgow, UK

2 The Oliver Zangwill Centre, Princess of Wales Hospital, Ely, UK

${ }^{3}$ Moss Rehabilitation Research Institute, Philadelphia, USA

${ }^{4}$ Department of Rehabilitation Medicine, Jefferson Medical College, Philadelphia, USA

The purpose of rehabilitation, including brain-injury rehabilitation, is to reduce disability and increase participation in valued activities. This broad purpose is reflected in the more specific aim of enabling people to achieve personally relevant goals. Thus systems of goal setting or goal planning are considered to be critical in the rehabilitation process. Several disciplines within psychology and medicine (but outwith brain injury rehabilitation) have elaborated theories related to goal attainment and self-regulation, the process of managing one's own goal-directed behaviour. These theories may be highly relevant to brain injury rehabilitation, with implications for maximising the achievement of goals during rehabilitation, being relevant to helping address characteristic deficits in executive function and teaching clients how to manage life tasks outside of formal rehabilitation. In this paper we review these theories and discuss reasons why theories of self-regulation and goal attainment are of particular relevance in brain injury rehabilitation. We outline some specific, testable interventions arising from the theories as well as their possible active ingredients, using illustrative examples from rehabilitation 
and other fields. Finally we consider a number of methodological issues relevant to testing hypotheses about goal-related interventions including appropriate experimental designs, participant samples and outcome measures.

\subsection{0-15.00: Community-Based Rehabilitation Following Traumatic Brain Injury (TBI)}

\section{J. Ponsford}

School of Psychology, Psychiatry and Psychological Medicine, Faculty of Medicine, Monash University, Melbourne, Australia

$\mathrm{T}$

There has been a growing awareness in recent years that many people with

TBI have difficulty in generalising what they learn in one situation to another and continue to experience numerous difficulties in their daily lives after leaving rehabilitation. The development of a community-based rehabilitation program will be described, which focuses directly on the roles and tasks relevant to the injured person in real work contexts, with active involvement of the injured person and close others. Results of a study evaluating 2-year outcome following this community-based intervention will be presented, comparing a group of 98 individuals with severe TBI who received community-based intervention with 98 matched TBI controls. Although earlier findings were less positive, following some revisions to the program, results indicate higher levels of independence in ADL in the group treated in the community, but no group differences in employment outcomes or emotional status. Costs, attendant care support and number of therapy sessions were lower for the community treatment group. The community group indicated a high level of satisfaction with the program. It would appear that implementation of community-based programs can be successful, although maximising contact with therapists and providing some interim contact with the centre seem important. 